\title{
La sociología en internet e internet para la sociología
}

\author{
Emilio Sáez Soro
}

\section{INTRODUCCIÓN}

En la última revisión de este artículo me di cuenta que la introducción ya era demasiado obvia para ser útil. Ya no necesitamos que nadie no «introduzca» en Internet, porque este mundo ya se encargó de rodearnos.

Internet está plenamente solidificado como ámbito de intercambio social de gran complejidad. Así, más allá de las teorizaciones acerca del Ciberespacio y sus cualidades específicas como contexto de acción social sumergido en «lo virtual» (Quéau, P. 1995), podemos observar como este entorno «normaliza» su presencia y se integra en otros procesos sociales. La red, no es un ente en sí, su entramado de millones de ordenadores conectados a través de los más variados sistemas de telecomunicaciones, no vale nada sí no es por los colectivos, las personas que instrumentalizan todo eso (Dyson, E. 1997:15). La idea de la red como algo importante y de gran potencial, lo es en tanto que es social.

Este objeto, debido a su complejidad socio-técnica resulta sumamente dúctil a la intervención en el mismo. Los usos y actividades que se desarrollan en este ámbito, evolucionan día a día en su estructura tecnológica y en la social, en la mutua relación que establecen, así como en los consensos y disensos que continuamente se producen. La gran variedad de actores que interactuan, así como la potencia inmensa de adaptabilidad del medio a las necesidades específicas de los mismos genera una gran riqueza de objetos. Realmente, del Internet de hace unos años al de ahora sólo quedan unas pocas cosas en común. La tendencia es hacía una evolución más rápida y sustancial, en la misma línea, cambios en la estructura tecnológica que permite la incorporación de nuevos colectivos de usuarios.

Esta maleabilidad, puede generar, de hecho lo hace, cierta confusión inicial. De todas formas, existen determinadas claves que clarifican las posibilidades y limitaciones de este medio para trabajar con él o para estudiarlo. 
Internet entra de lleno en la lógica de una economía de mercado, de una sociedad que se organiza como una red de intercambios de alcance mundial. Esta forma de organización requiere de un enorme y complejo sistema de comunicación. Internet, es así, una red de ordenadores con sus usuarios y organizaciones; de un enorme tamaño y dinamismo. De alguna forma, Internet es un objeto social que nos sirve a los sociólogos como laboratorio de observación de lo que es la realidad social mundial en extenso. El hecho de que en esta red de comunicación e información los emisores sean potencialmente tantos como los receptores, hace que la diversidad en las interpretaciones de los distintos temas tratados pueda parecerse mucho a la que existe en el contexto concreto.

La visión de este objeto se ha de considerar desde su componente "vivo", la dinámica social que se desarrolla en su seno y en torno a el. Es imprescindible considerarlos así, porque luego encontraremos que dicho dinamismo hace que nada sea inmovil en este mundo y que en cada mirada todo cambie. Para aprovechar la información, las capacidades comunicativas, etc. es necesario comprender que dicha información, que una realidad concreta, se transformará ante nuestros ojos o detrás de ellos con gran rapidez.

Podemos observar comunidades que sólo tienen sentido en este contexto (Rheingold, H. 1996: 413), o que teniendo otros motivos de formación, es la facilidad de comunicación que ofrece Internet lo que las hace posibles.

Un tercer elemento de interés para la sociología es el uso del «objeto» de Internet como un comodín todo terreno en los discursos más pretenciosamente modernizadores. De alguna forma Internet, como tal, trasciende las simplificaciones ya que en su seno se dan grandes dosis de contradicción y conflicto, siendo su complejidad tanto interna como externa un reflejo de la sociedad que lo participa ${ }^{1}$.

Para comprender mejor este mundo hemos de mirarlo con un marco amplio. Es un entorno complejo y variado de matices, tanto en la comunicación que se desarrolla, en la información que se almacena, como en las comunidades y grupos que se forman a través de este medio. Las posibilidades que tenemos de poder usar todo ésto o de buscar objetos dignos de ser investigados, prácticamente tiene los mismos límites que nuestra imaginación.

\section{INTERNET COMO HERRAMIENTA PARA LA INVESTIGACIÓN SOCIOLÓGICA}

Internet ofrece multitud de posibilidades para el trabajo habitual de la investigación sociológica. Son dos los principales capítulos los que agrupan las

1 Aunque es necesaria la referencia a Internet como objeto social, no es éste el principal interés del presente artículo. En este sentido y respecto a la bibliografía, es muy numerosa la relacion de títulos dentro de lo que viene a llamarse Cibersociología o Ciberteoría. Sin embargo no conozco títulos que traten de la red Internet como herramienta metodológica. De forma ilustrativa, al final del artículo adjuntaré una selección de bibliografía en español para la comprensión de Internet como objeto social. 
utilidades más específicas, éstos son los de fuente de información y el de herramienta de comunicación. Otro aspecto menos explícito pero pero más interesante es el de ser un observatorio privilegiado para muchos fenómenos que se manifiestan allí.

Estas tres utilidades se entremezclan en multitud de ocasiones. Hay que tener en cuenta que las herramientas que se diseñan para funcionar en Internet son tan potentes y flexibles como las propias tecnologías que las sustentan. La capacidad de refinar, segmentar las funciones que han de cumplir estos artefactos llegan a la personalización de los mismos. La combinación de la tecnología de proceso computacional de datos junto con la de telecomunicaciones, con el añadido del establecimiento de grandes redes supone más que un objeto con el que hacer cosas, un mundo peculiar donde podemos adaptar las tecnologías a nuestras necesidades específicas.

En este sentido, a la hora de abordar el uso de las distintas herramientas que nos proporciona la red Internet es necesario plantearse la combinación de distintas herramientas ya sea de suministro de información, de comunicación, para satisfacer nuestras necesidades. Lo más probable es que generemos usos específicos para nuestro trabajo preparando herramientas infocomunicativas originales y adecuadas a un fin.

Todas esta herramientas están sometidas a la obsolescencia propia de los artefactos informáticos, en su formato de programas o en las máquinas. Ésto puede suponer un quebranto de los ritmos habituales de trabajo para asimilar las nuevas cualidades de las recién incorporadas herramientas, con la agravante de que dada la mayor complejidad cuesta un esfuerzo añadido poder controlarlos.

Nos encontramos ante una especie de maldición, por una parte no podemos dejar de usar estas herramientas, ya que nos ofrecen posibilidades muy valiosas tanto en el ámbito de la comunicación como en el de la información. Una vez inmersos en este mundo con sus «artefactos» ya no podemos dejar de evolucionar con el mismo, éste nos arrastra con sus rápidos desarrollos.

A los que nos vemos obligados a entrar de pleno en este mundo, nos queda el consuelo de que con la popularización de este medio se facilita el uso de los nuevos artilugios.

Este proceso de seguimiento tecnológico tiene importancia, ya que si las relaciones sociales que se producen en el Ciberespacio no cambian, sí que lo hacen en las formas de manifestación, por lo que es necesario la comprensión de estos mecanismos para realizar un correcto seguimiento.

\subsection{Herramientas de acceso a información de interés sociológico}

La gran cantidad y variedad de información que podemos encontrar en Internet es muy útil para la sociología en dos aspectos: para obtener información específicamente sociológica, además de abundante material secundario.

Sin embargo, esta abundancia conlleva ciertas dificultades. El desorden, la difícil localización, la heterogeneidad formal y la propia irregularidad en la 
calidad de los contenidos, hacen que el aprovechamiento de esta fuente de información sea compleja.

Estas distintas fuentes de información pueden ser de gran utilidad si las combinamos para ilustrar un mismo tema. Para ello, tenemos que manipular esta información de dos formas. Por un lado, la información obtenida de organizaciones a través de estadísticas, documentos, etcétera, no requiere un tratamiento especial. El otro tipo de información suministrada por los propios colectivos objetos de investigación, es necesario situarla en un contexto específico en el que se mezcla la situación que le es propia a cada colectivo concreto y, además las características del medio en el que se manifiestan, dícese Internet.

Está diferenciación resulta importante porque los interlocutores tienen nuevas posibilidades de expresión que les permiten generar «objetos» de comunicación e información sustancialmente distintos de los hasta ahora existentes. En este sentido, me refiero a las posibilidades que aporta el lenguaje hipertexto para generar discursos colectivos, así como todas las posibilidades programáticas para el establecimiento de procesos info-comunicativos en distintos ritmos temporales.

Estas nuevas realidades de la expresión colectiva plantean problemas para su estudio pero a su vez son un estímulo para la investigación metodológica en ciencias sociales, así como para la comprensión de la compleja comunicación humana.

Para acotar el interés de este trabajo voy a definir un marco hipotético, desde un objetivo bastante amplio: el de establecer pautas de trabajo sencillas y formalizadas para aprovechar los materiales informativos que aporta la red Internet a la investigación sociológica.

Las hipótesis que se han de cumplir para alcanzar este objetivo:

- Qué el uso de la red Internet como marco de observación de fenómenos sociales externos o internos a la misma, será representativo de los mismos siempre que se den unas condiciones de complejidad y tiempo de observación suficientes definidos por su propia naturaleza.

\subsubsection{Búsquedas de información}

Buscar la información en Internet puede ser una tarea compleja. Esta complejidad se concreta más por el ruido que recibimos, próximo al objeto de nuestro interés que por la falta de información.

Esta problemática se desarrolló al mismo tiempo que crecía telaraña informativa del web y que se creaban herramientas de búsqueda informativa. El problema, reside en que a pesar de que estás herramientas registran decenas de millones de páginas informativas, no hay ninguna que pueda presumir de que controlar todo el contenido informativo de la red. Por una parte, dichas herramientas nos pueden llegar a ofrecer miles de páginas supuestamente 
relacionadas con nuestro objeto de interés y de otro lado ni siquiera podemos estar seguros de que ahí está lo más importante. Resulta significativo que en distintos buscadores (pongamos Lycos y Altavista) las primeras páginas por orden de relevancia sobre un tema en el que hemos efectuado una orden de búsqueda, sean totalmente distintas y es posible que las que aparecen en un buscador como más importantes no lo hagan en ningún término en el segundo.

En ese sentido, siempre es necesario combinar varias herramientas buscadores si queremos tener mayores posibilidades de encontrar lo que deseamos. $\mathrm{Y}$ al final, a pesar de todo, siempre nos ha de quedar la incertidumbre de que seguramente nos dejaremos algo importante.

Un último paso para hacer más eficaces las herramientas de búsqueda de información ha sido generar instrumentos especializados. De esta forma, tenemos buscadores de temas económicos, de temas legislativos, de direcciones de correo y por supuesto de temas de interés sociológico.

De esta forma el número de herramientas buscadoras de información en Internet, crece día a día. Aunque esto supone mayores posibilidades de localizar lo que buscamos, por otra parte lleva hacia una abundancia desmesurada de información relacionada con nuestro objeto de búsqueda. Conseguimos información más cercana a nuestras necesidades pero es difícil sustraerse a la invasión en cantidad de la misma.

Nos podemos ver inundados si buscamos información de forma genérica con un objeto concreto que pretendamos investigar. Ya que nos podemos ver inundados por referencias de cientos de miles de documentos que poseen alguna información relativa a nuestra petición. Es en este tipo de experiencias donde observamos con claridad la necesidad de concreción en las solicitudes de información. Nos vemos obligados a dividir el objeto sobre el que pretendemos trabajar en el mayor número de partes posibles e indagar de forma especializada.

Esta problemática nos hace ver la vitalidad y dinamismo de esta red social llamada Internet. De esta forma, siempre que busquemos algún tipo de información y queramos llegar a conocer todo lo posible es conveniente, además de utilizar las herramientas buscadoras, podremos echar mano de los grupos organizados respecto a un tema específico.

Internet puede ser una herramienta de comunicación e información. La parte de la comunicación es una fuente estratégica de datos inigualable. El hecho de que millones de usuarios de la red Internet se organicen en foros formal y temáticamente distintos ${ }^{2}$, permite acudir a ellos para solicitar información acerca de los elementos sobre los que se esté discutiendo, conversando o trabajando. Es la especialización de algunos de estos foros lo que nos puede ofrecer una mayor especificidad y actualización sobre la información que nos sea de interés.

${ }^{2}$ Mis estimaciones personales sobre la evolución del número de grupos de discusión y trabajo en Internet son de una multiplicación por cuatro en los últimos tres años, llegando en estos momentos a una cifra aproximada de cien mil grupos temáticos distintos. 


\subsubsection{Centros de información sociológicos o de interés para la sociología}

Por supuesto la sociología tiene su lugar como disciplina específica en Internet. De esta forma multitud de organizaciones gubernamentales, universitarias, privadas, etcétera, tienen un fondo de información y comunicación a través del web en Internet.

Dichas sedes virtuales, suponen un importante avance para el servicio que prestan al investigador social. Se agiliza la difusión informativa, distribuyéndose documentación específica con enorme facilidad y se mejoran e incrementan las comunicaciones y contactos de especialistas de distintos países.

Este último punto es de especial relevancia, ya que se facilita el contacto y la comunicación entre investigadores de materias próximas. Localizar a investigadores de materias de nuestro interés es muy sencillo revisando la información de los departamentos de otras universidades, fondos de información sobre dichas materias, listas o grupos de noticias que trabajen sobre dicho asunto, etc. Desde que se ha generalizado el uso de la red en el ámbito de la investigación es muy fácil estar pendiente de lo que se trabaja en el tema que nos compete al otro lado del mundo.

A este respecto resultará clave, no sólo el hecho de poder comunicarnos con otros colegas sino que además éstos tengan interés por hacerlo, algo que quizás se da demasiado por sentado pero que a la hora de la verdad puede resultar bastante complicado. En este medio es donde las fronteras culturales se hacen más evidentes.

El establecimiento de sedes web correspondientes a las organizaciones tradicionalmente distribuidoras de información útil para la investigación social suponen una revolución real en las posibilidades de acceso a todo tipo de documentos. La riqueza de los formatos en los que se puede plasmar la información, sonido, textos, gráficos, vídeo, así como formatos «dinámicos» que se generan a partir de las propias tecnologías de soporte. Estoy hablando de hipertexto, Java, así como otros programas que se incrustan entre la información dándole a la misma un gran dinamismo para su mejor aprovechamiento.

Las organizaciones vinculadas con la investigación pueden aprovechar esto medios para difundir su información de forma muy eficaz.

La capacidad para discriminar el acceso y la sencillez de transferencia resultan claves para estos fines. A pesar de que la difusión informativa no está generalizada por estas vías, será el destino definitivo para el acceso a los bancos de información por razones contundentes de eficacia y eficiencia.

$\mathrm{Si}$ añadimos la facilidad que supone este medio para acceder a recursos informativos en otros países es de prever que se avance de forma significativa hacia lo que podríamos denominar una actualización permanente del «estado de la cuestión» científica.

Este proceso significaría una necesaria homogeneización de criterios tanto en la cantidad como en la cualidad de la información ofrecida, así como una «nivelación» cultural para lograr el acceso universal a toda la información generada. De alguna forma la principal barrera hacia la consecución de un acceso universal y homogéneo a la información producida se encuentra en la propia 
idiosincrasia cultural del contexto donde se genere. La cuestión idiomática es la más importante más allá de formatos de informática varia. Es claro que el ingles lleva mucha delantera en este terreno. El resto de los idiomas tienen una presencia menor. Son muchos los investigadores de paises no angloparlantes que sitúan en la red sus artículos y materiales directamente en ingles, pero esta práctica no es generalizada y no es previsible que lo sea a corto plazo, al menos en España, donde casi nadie publica en ingles a través del web.

\section{2. «Colgando» herramientas en la red}

Colgarnos de la red y observar, medir, tentar al experimento y tantas otras cosas, pueden ser una vía de aproximación para usar métodos viejos y correr con otros nuevos.

Respecto a las viejas, ya existen múltiples experiencias de encuestas a través de Internet ${ }^{3}$. En este terreno las encuestas realizadas, lo han sido a través del web, con la invitación al «visitante» de que cumplimentase las preguntas y a través del correo electrónico, con cuestionario adjunto. Otras modalidades más desarrolladas son las que invitan a través de correo electrónico a responder a una encuesta situada en una página web. De esta forma, sí el encuestad no tiene interés en responder se evita la transferencia del cuestionario.

Como resulta evidente, el principal problema del diseño de encuestas a través de estos procedimientos es el de la falta de muestras de calidad o suficientemente controladas.

Resulta muy interesante la posibilidad que ofrecen los medios de conversación en tiempo simultaneo (tipo IRC, multiconferencia, etc.) para la realización de entrevistas en profundidad y grupos de discusión. En este caso el empleo de estos medios puede ahorrar mucho tiempo y dinero en la realización de entrevistas. Se evitan desplazamientos, alquileres de espacios y transcripción de grabaciones. Por otra parte, es necesario adaptar las variaciones en la forma del discurso, ya que es muy difícil que en la entrevista escrita en tiempo simultaneo se produzca la discursividad propia del dialogo oral.

Esta posibilidad resulta interesante para investigaciones de colectivos dentro de la propia red; por dos motivos, uno que supone la posibilidad de entrevistar a a los «actores» en el medio donde se desenvuelven de forma armónica y la segunda, que al ser ese medio deslocalizado, muchos de estos actores están situados en zonas muy distantes.

Por otra parte la red proporciona abundantes y heterogéneos materiales producidos por múltiples actores sociales. Podemos generar con sencillez colecciones de documentación digital en relación con cualquier objeto social.

La introducción del hipertexto y de su complemento, el hipermedia, es un salto cualitativo en la distribución informativa. Esta integración de información

${ }^{3}$ http://www.umich.edu/ sgupta/hermes/ y las encuestas de usuarios de Internet en España del EGM: http://www.egm.es 
y comunicación con distintos formatos (texto, imagen, sonido, vídeo, constructos programáticos interactivos, etc.) y la estructuración en red, interna y externa, a través del hipertexto, nos sitúa ante un nuevo objeto que merece ser tratado de forma específica.

El hipertexto como objeto, es el alma del web y éste a su vez, es el cuerpo de Internet. Hay que desarrollar por su interés, métodos que aprehendan la naturaleza relacional e integradora del hipertexto al estudiar los contenidos y continentes de páginas web interesantes para la sociología.

\section{EL OBSERVATORIO}

No se ha de perder de vista que Internet para la sociología, además de ser una fuente «formal» de acceso a la información es también un objeto social con su propia dinámica y que en muchos aspectos supone un auténtico objeto de estudio en sí.

Podemos hacer una distribución inmediata respecto a la actividad social que se genera en Internet: por una parte tenemos la actividad que es específica y exclusiva de la red y que no se daría fuera de ella y por la otra, Internet supone un escaparate de los movimientos sociales que intentan hacerse ver a través de este medio, generando información y comunicación de muy diverso tipo.

\subsection{Manifestaciones formales}

Las organizaciones formales han comenzado a utilizar Internet. Para sus fines de información y comunicación desde el primer momento. No hay que olvidar que Internet tiene su origen en una iniciativa institucional, a través del ejército y la universidad de los Estados Unidos.

De forma progresiva y muy rápida en los últimos tiempos las organizaciones formales, tanto privadas como públicas se han ido incorporando a este medio. En un primer momento se distribuía información corporativa. Esta función inicial se diversifica: recepción de sugerencias, petición de informaciones específicas, tramitación administrativa , etcétera.

Esta aparición de organizaciones formales en Internet puede ayudar a conseguir sus fines pero está muy lejos de haberse desarrollado en todo su potencial. La facilidad con la que podemos acceder a toda esta información supone un acercamiento a determinados aspectos informativos que anteriormente eran muy costosos de conseguir.

\subsection{Manifestaciones informales}

La facilidad con la que se puede publicar información en Internet ha supuesto para muchas pequeñas organizaciones, tener una vía muy efectiva de difusión de sus actividades. 
Es fácil de imaginar que los más activos en Internet serán los más reivindicativos. Señalar el hecho de que Internet sirve en numerosos casos de «argamasa» para generar movimientos reivindicativos que de otra forma no llegarían a alcanzar la suficiente «masa crítica». Además se producen movimientos sociales de reivindicación «ciberendógenos», que se constituyen alrededor de los propios conflictos del Ciberespacio: movimiento en pos de la tarifa plana, movimiento contra la censura en Internet, movimiento contra la pedofilia en Internet, movimiento por un sistema operativo informático universal y gratuito, etc.

El problema de este tipo de organizaciones y movimientos es que no tienen un presencia sólida en la red, su falta de infraestructura les dificulta realizar una labor comunicativa con una proyección temporal significativa. Lo herrático y temporal es habitual en la presencia de estas organizaciones.

\subsection{Acontecimientos}

Con las fuentes de información señaladas podemos seguir cualquier evento informativo que se produzca en el mundo con bastante detalle. A ésto hay que sumar la riqueza de fuentes del medio que nos puede dar una comprensión del fenómeno observado bastante rico en detalles. De alguna forma la documentación sobre los temas de actualidad se enriquece en formatos y voces.

Merece la pena resaltar el hecho de que a diferencia de otros medios informativos, ya sean oficiales o extraoficiales en Internet, por lo general, la fuente es accesible de forma casi inmediata a través del correo electrónico, lo que permite un mayor acercamiento al motivo de nuestro interés. De alguna forma todos podemos ser emisores en la red y la generación de interlocutores informativos respecto determinados temas se multiplica.

\section{GUf́A DE RECURSOS (NADA SISTEMÁTICA) PARA HINCAR EL DIENTE}

Puede ser útil proponer lugares de interés general que nos puedan dar una idea de cómo se estructuran y desarrollan los contenidos de interés para la sociología en Internet. Dadas las características de este medio, es absurdo pretender hacer una «colección definitiva», entre bajas y altas, además de los que nunca se encuentran pero sabemos o intuimos que están, siempre se estará con la incertidumbre de que hay algo más....con lo cual, inevitablemente restamos valor al esfuerzo realizado. No hay que preocuparse demasiado del estado de acumulación de recursos, sino que lo más útil es tener claras ciertas pautas de búsqueda y ciertos lugares clave desde donde se abren los mejores caminos para llegar a lo que necesitamos. 


\subsection{Buscadores e índices}

A pesar de que existen buscadores específicos para determinadas materias, lo más normal es empezar una búsqueda por herramientas genéricas. Respecto a este tipo hay dos directrices básicas, para búsquedas de ítems muy específicos es mejor recurrir a las máquinas buscadoras. Éstas desde sus fondos de millones de páginas extraídas del web nos suministraran las direcciones que contienen los elementos que pidamos con la precisión que nos permitan sus herramientas de búsqueda. Para búsquedas más genéricas recomiendo los índices, ya que en los mismos encontraremos sistematizada información relativa al asunto que tratemos.

En cuanto a buscadores, el más prestigioso es en estos momentos "Altavista», por una razón tan sencilla como la de ofrecer los mejores resultados en el menor tiempo.

La sede americana que es la más completa está en:

Http://altavista.digital.com

La española:

Http://altavista.magallanes.net

Una forma muy práctica de acceder a estos buscadores es a través del mejor índice: Yahoo. Ésta colección de recursos indexados es en estos momentos la mayor del mundo, además contiene una pasarela directa a Altavista, de tal forma que cuando no encontramos algo en este lugar nos desvía directamente la búsqueda para obtener resultados de Altavista. De esta forma u obtenemos los resultados de las búsquedas sistematizados en los índices temáticos, con referencias paralelas al mismo tema o directamente nos conduce a un material más diverso producido en una busca abierta en el universo web.

Otra forma muy efectiva de obtener buenos resultados en nuestras búsquedas de información en Internet, es la de usar «metabuscadores», es decir buscadores que realizan su consulta en varias máquinas o servicios a la vez y devuelven los mejores resultados de cada una (normalmente) unificados en un listado.

Quizás el mejor de todos sea:

\section{http://www.metcrawler.com}

Este metabuscador ofrece resultados de Altavista, Yahoo, Webcrawler y Lycos, así como de otros de los mejores buscadores.

Sí tenemos claro el ámbito geográfico donde está la información que queremos es mejor utilizar los buscadores e índices de ese lugar ya que por motivos obvios será más fácil localizarla.

En España existen ya numerosas herramientas para estos fines. Tenemos algunos índices, como:

http://www.ole.es

http://www.ozu.es 
Todas estas herramientas de localización de información se han convertido en la pieza clave para que Internet sea algo útil. Por otra parte son en estos momentos y con diferencia el mejor negocio de todos los que han surgido en este contexto, a través de la publicidad que aparece cuando se realizan las búsquedas. Este hecho ha llevado a la aparición de cientos de estos servicios en toda la red y por supuesto, la competencia entre los mismos. Todo este proceso ha conducido a un incremento de servicios asociados a la herramienta de búsquedas unido a lo que casi es la garantía de la gratuidad de un servicio que es esencial para el aprovechamiento de Internet. Entre los servicios gratuitos podemos encontrar algunos realmente útiles, entre ellos servicios de traducción entre varios idiomas y el ingles (Altavista), pasarelas especializadas para la localización de material muy específico (imágenes, software, sonidos, etc.) y el que resulta de mayor utilidad, servicios de personalización de la búsqueda informativa. Estos últimos servicios nos dan la posibilidad de realizar las búsquedas en un entorno «personal» de tal forma que ese espacio informativo se impregna de nuestras preferencias habituales en el momento de buscar información y comienza a generar sus propias búsquedas en esas materias para nosotros.

Este tipo de servicios personalizados es el futuro más próximo de los buscadores de información ya que en ellos se concentra de forma sustancial grandes utilidades con ahorro de tiempo y acceso a informaciones de interés para el usuario, además de la otra cara de la moneda: se establecen perfiles detallados de los usuarios, aprovechables para generar publicidad con una segmentación exquisita.

Tenemos un ejemplo práctico de este tipo de utilidades en Yahoo:

http://edit.my.yahoo.com/config/login

Por otro lado podemos utilizar también software de búsquedas personalizable, que residente en nuestro propio ordenador extrae de múltiples buscadores de Internet la información solicitada almacenándola en nuestro disco duro. Ya existen diversos programas de este tipo que se pueden usar gratuitamente durante un tiempo determinado. Quizás la mejor utilidad de este tipo de herramientas es que pueden estar trabajando mientras nosotros no estamos al ordenador buscándonos la información que se ciña a los parámetros definidos. No son demasiado útiles estos programas cuando sólo contamos con una conexión telefónica, ya que son bastante lentos y pueden consumir muchas horas de conexión. Un buen ejemplo de este tipo de software lo podemos conseguir en:

http://www.agentware.com

Por último queda mencionar las propias guías o compendios de direcciones en papel. Las guías clásicas que pueden ser de utilidad cuando están muy especializadas, sobre todo por la comodidad que siempre supone usar una buena guía en papel. Sus desventajas se encuentran en la rápida obsolescencia a la que se ven sometidas, que en algunos casos, sobre todo en las obras no especializadas, es tan evidente que cuando salen a la venta ya dejan de tener utilidad. 


\section{2. Recursos sociológicos}

El acceso más claro y directo a fuentes de información específica para la investigación sociológica se encuentra en los propios directorios realizados al efecto en centros relacionados con la sociología. Comienza a existir un gran número de recursos tipo directorio. En este sentido y siguiendo la filosofía de intentar establecer los referentes más útiles por su potencia y claridad recomiendo un directorio de alcance mundial que es magnífico en todos los aspectos. Me refiero al directorio Sociosite de la Universidad de Amsterdam,

\section{http://www.pscw.uva.nl/sociosite/}

En este lugar encontramos información abundante sobre todas las referencias sociológicas imaginables con la descripción de cada lugar. Por otra parte, la ordenación de dichas referencias es muy adecuada, contando con un útil buscador interno para localizarlas más rápido.

De alguna forma, conociendo lugares como el Sociosite, es inútil realizar esfuerzos paralelos de recopilación a no ser que tengan una visión muy especializada o muy de atajo, ya que la exhaustividad de dicho lugar al tratar todos los temas es ejemplar.

Siguiendo con la idea anterior de mencionar recursos más próximos geográficamente, no puedo citar en España ningún directorio de recursos sociologicos «modélico». En realidad, directorios de recursos generales no hay a fecha de hoy ninguno en este país que pueda considerarse interesante. Desde una perspectiva de mayor especialización sí que resultan de interés algunos directorios estadísticos y de referencia a bancos de datos. Así tenemos como más útiles los del CIS y el INE.

http://cis.sociol.es

http://www.ine.es

Desde éstos se puede acceder a multitud de otros servicios de publicación de datos estadísticos y resultados de encuestas.

Por otra parte se pueden acceder a listados de recursos genéricos sobre sociología en España a través de los propios índices generales. Aunque dichos índices no tienen una información de calidad suelen aparecer bastantes de las referencias existentes. Una vía más para acceder en España a recurso de información sociológica son los listados que ofrecen los distintos departamentos universitarios españoles de sociología, aunque estos suelen adolecer de poca actualización de contenidos. Es de esperar, que en breve tiempo se tengan más en cuenta este tipo de detalles.

\section{3. Observatorios}

Como ya comenté, Internet puede ser un lugar desde el que observar de forma activa o pasiva, distintas dinámicas sociales, con el añadido de que esa 
observación puede generar automáticamente los materiales que la describen. En este sentido tenemos dos modalidades de observatorios, los que podríamos llamar estáticos y los dinámicos. Con los primeros hago referencia a las páginas web generadas por los grupos que quieren comunicarse y que se van actualizando a modo de publicación. Los dinámicos son los debates, los lugares de interacción comunicativa en tiempo simultaneo o casi simultaneo.

Respecto a las páginas web de colectivos concretos siempre podremos localizarlas con las pautas habituales de uso de buscadores e índices. No haré aquí un listado de direcciones ya que los distintos motivos de interés pueden ser tan diversos que carecería de sentido. Sí que hay que contar con este tipo de información cuando pretendemos seguir un determinado objeto social, porque es algo, bastante más que el material publicado en papel, ya que normalmente se suelen recoger apartados para el debate casi en vivo y suelen facilitar medios para ponerse en contacto con los integrantes del grupo u organización. De esta forma las páginas web nos proporcionan un acceso a la información generada por un grupo formal o informal, el seguimiento de debates generados en ese contexto, así como una vía rápida y eficaz de contacto con sus integrantes.

El componente «activo» de la red tiene varias partes que se diferencian en la forma más que en el fondo. El fondo consiste en una conversación grupal sobre un tema más o menos concreto. La gracia del asunto está en la facilidad con la que varios interlocutores situados en cualquier parte del espacio pueden compartir un debate que les une.

Existen tres tipos de foros: los grupos de noticias (coloquialmente «las news"), las listas de correo y el IRC (o chat). En principio estas tres modalidades pueden tener un fin muy similar: favorecer debates o conversaciones entre grupos de personas con intereses comunes.

Podemos diferenciar estos medios respecto a las dos variables mencionadas: las listas de correo son un medio al que accedemos cuando lo hacemos al correo electrónico, ya que los mensajes que se generan aparecen mezclados con el correo electrónico convencional. Estos mensajes son enviados por un redistribuidor común a todos los integrantes de la lista, a la que hay que suscribirse para recibir dichos mensajes. De esta forma tenemos un acceso a la información normalmente en tiempo diferido, de una o dos veces al día, que son las pautas habituales de acceso al correo electrónico. Luego, el acceso a esta información es indiscriminado, porque habitualmente volcaremos en el buzón de correo todos los mensajes generados por la lista. El hecho de que sea necesario inscribirse en estos grupos y que además se reciban indiscriminadamente todos los mensajes producidos en la misma, produce un notable control interno que hace que estos foros sean los más «serios» de todas las modalidades que hay.

Los grupos de noticias son tablones electrónicos donde se envían mensajes relacionados con el tema de cabecera. En este caso hay que acudir a los mensajes para poder leerlos, también en diferido. Lo usual es acceder al listado de mensajes generado por orden cronológico de aparición de los mismos. El listado de mensajes aparece con información de referencia sobre su contenido y autor, de tal forma que podemos elegir que mensajes queremos leer y cuales no, 
sin necesidad previa de cargarlos al ordenador. Esta capacidad del lector de una mayor discriminación del contenido de estos grupos relaja más el control de lo que se vierte en dichos foros. Ésto es evidente si vemos la enorme cantidad de spam ${ }^{4}$ que se genera en estos grupos, algo que en las listas de correo está severamente penalizado.

Los grupos de conversación que se generan en el entorno del IRC ${ }^{5}$ tienen un acceso a la conversación ligado a la presencia de sus contertulios. Las conversaciones que se desarrollan en estos foros o canales suelen tener un tema que da marco a las mismas. Sin embargo, en este medio hay una tendencia muy predominante a lo lúdico. Los canales suelen ser abiertos y la entrada y salida constante de integrantes del foro dificulta mantener el hilo de una conversación habitual si es que hay alguna. Luego hay que añadir el hecho de que muchos de estos canales se usan más que como lugar de encuentro para entablar conversaciones privadas que como foro organizado de conversación.

Creo que es interesante anotar que entre listas de correo y grupos de noticias, se recogen unos cincuenta mil foros distintos, aunque en muchos casos puedan tener temas similares de discusión. $\mathrm{Y}$ es digno de consideración, el hecho de que cualquier usuario de Internet tiene libre el acceso potencial a los mismos.

A continuación ofrezco una muestra de los distintos foros mencionados anteriormente, por el interés que puede tener tanto conocer la composición de los mismos como la existencia de grupos que merezca conocerse en sí.

\section{Grupos de NEWS españoles}

Estos son algunos de los grupos españoles de news, sus temáticas son muy diversas y van creciendo paulatinamente en cantidad y diversidad. Dichos foros se crean a partir de la petición de un número mínimo de usuarios interesados (alrededor de veinte personas) y pueden ser abiertos o moderados, aunque esta última posibilidad escasea en los grupos de noticias.

La temática de los mismos es muy variada, teniendo especial protagonismo los asuntos relacionados con el mundo de la informática y otros que se generan desde la universidad. Por supuesto cualquier usuario de Internet en España puede acceder a grupos internacionales o de otros países y participar en los mismos de forma activa o pasiva.

${ }^{4}$ Con la palabra spam, que su traducción literal sería algo así como carne de cerdo enlata$\mathrm{da}$, se hace referencia a los mensajes que se envían a multitud de grupos de noticias o listas de correo de forma simultanea y que además, no suelen tener relación con los temas de dichos foros. Esta práctica está muy mal vista por la mayoría de usuarios de la red Internet pero se está generalizando su uso como formula barata de hacer mailings publicitarios masivos.

5 Las siglas IRC, hacen referencia al Internet Relay Chat, que podría traducirse por «transmisión de conversaciones en Internet». Este servicio de Internet consiste en la conexión a máquinas servidoras que hacen de nexo común entre varios usuarios para mantener una conversación por escrito en tiempo simultaneo. 
Los programas que nos permiten leer estos tablones electrónicos ${ }^{6}$ nos ofrecen un listado de los mensajes con distintos criterios de ordenación (fecha de llegada, nombre del emisor, título, etc.) además de ofrecer la opción de «encadenar» los debates. Es decir podemos ver distintas cadenas de mensajes que están relacionados con un tema de discusión concreto y al que responden los interesados. De esta forma al cargar el listado de mensajes de un grupo podemos observar en numerosas ocasiones como existen agrupaciones de los mismos respecto a un título concreto de discusión.

En grupos con cierta tradición, se establecen mecanismos de relación que se estructuran sobre intercambios de la más variada naturaleza. En este sentido, podemos observar en muchos de estos grupos que existe una distribución de roles que en ocasiones es de gran complejidad.

En este caso, normalmente las pautas de liderazgo de los grupos se establecen alrededor del conocimiento del tema tratado. Al ser la mayoría de estos foros monotemáticos provocan con el tiempo una gran especialización de los debates que necesariamente conlleva a que los «gurús» del asunto sean referencia obligatoria para los lectores del mismo.

Aunque la calidad de los contenidos que se vierten en estos foros es bastante heterogénea, en multitud de ocasiones podemos llegar a conocer determinadas informaciones sumamente valiosas respecto al tema tratado. En este sentido, hay cierta polémica en el seno de algunos de estos grupos, respecto a la "vampirización» de ideas e información por terceros que leen pero no aportan nada a dichos grupos. Algunos usuarios de países anglosajones critican a los foros de discusión de fondo cultural latino por ser menos participativos y más egoístas las actitudes de sus integrantes. De todas formas, es un estudio por hacer ya que a veces tampoco es la cantidad lo más importante...

En el listado que podemos ver a continuación están casi todos los grupos de discusión registrados en España ${ }^{7}$. Los nombres de los mismos son lo suficientemente explícitos como para comprender su temática, de todas formas la mejor comprensión de su funcionamiento y contenidos se consigue explorándolos.
es.alt.anuncios
es.alt.binarios.astronomia
es.alt.binarios.sexo
es.alt.chistes
es.alt.sexo
es.charla.actualidad

${ }^{6}$ Existen multitud de programas gratuitos para poder acceder a estos tablones electrónicos: Netscape e Internet Explorer (en sus partes dedicadas a éstos temas) y FreeAgent como programa específico para la lectura de news.

7 Del listado eliminé los grupos más redundantes, por otra parte hay que tener en cuenta que estas listas dependen también del servicio que ofrece cada proveedor de Internet. Ya que lo más habitual es que no todos los proveedores, públicos o privados, ofrezcan todos los grupos. De alguna manera los administradores de los servicios «seleccionan» cuales son los grupos a los que los usuarios acceden y a los que no acceden. 
es.charla.conexion.tarifa-plana

es.charla.cooperacion

es.charla.economia

es.charla.economia.bolsa

es.charla.educacion

es.charla.educacion.distancia

es.charla.enfermedad.cancer

es.charla.enfermedad.diabetes

es.charla.gastronomia

es.charla.gay-lesbiana

es.charla.medio-ambiente

es.charla.moteros

es.charla.motor

es.charla.politica

es.charla.religion

es.charla.sindical

es.charla.utopia

es.ciencia

es.ciencia.astrofisica

es.ciencia.electronica

es.ciencia.enologia

es.ciencia.matematicas

es.ciencia.medicina

es.ciencia.quimicas

es.comp.artes-graficas

es.comp.demos

es.comp.emuladores

es.comp.hackers

es.comp.hardware

es.comp.infosistemas

es.comp.lenguajes.c

es.comp.macintosh

es.comp.os.ms-windows

es.comp.seguridad.pgp

es.comp.sistemas.inteligentes

es.comp.virus

es.humanidades.derecho

es.humanidades.gramatica

es.humanidades.literatura

es.humanidades.psicologia

es.news

es.news.preguntas

es.pruebas

es.rec.aviacion

es.rec.cine

es.rec.comics

es.rec.deportes

es.rec.deportes.atletismo

es.rec.deportes.aventura

es.rec.deportes.baloncesto 




\section{Grupos de IRC del canal hispano}

Sigo al igual que en los grupos de noticias, describiendo foros que se encuentran inmersos en el contexto cultural de España, aunque siempre abiertos al acceso de visitantes de cualquier otro país del mundo.

Los grupos de IRC son los más dinámicos de todo Internet, también es posible que sean los menos prácticos para un uso serio. El caso es que una visita a estos «canales» de comunicación resulta siempre entretenida.

Esta modalidad de comunicación, tiene dos partes claramente diferenciadas. La parte más clara y explícita es la comunicación pública que se produce en los canales de acceso libre entre multitud de personas (ver listado). La otra parte, incluso más activa, es la de las conversaciones privadas que se mantienen entre dos personas de forma exclusiva o paralela a otro tipo de conversaciones ya sean privadas o en canales públicos. Estas dos modalidades están muy integradas, siendo habitual que los componentes de un canal público tengan dos niveles de conversación entre sí. 
A su vez, existen dos tipos de canales de conversación, los permanentes, que están abiertos las veinticuatro horas del día y los provisionales que son coyunturales a un tiempo determinado. En los canales permanentes, se establece al igual que en otro tipo de foros toda una micro estructura social de funcionamiento, pero en este caso se añade una superestructura técnica que explicita las jerarquías. De esta forma, un programa asociado a éste mecanismo de comunicación permite estructurar unos niveles jerárquicos de privilegios para el control del canal.

Esta descripción de mecanismos de control parece un poco exagerada para algo que en principio no es más que un medio de comunicación. Pero cuando entramos en un canal en el que hay cerca de cien personas charlando, es algo más que un medio de comunicación y dependiendo de la naturaleza de dicho lugar, podemos encontrar pautas de relación sumamente conflictivas que se resuelven por las vías más expeditivas ${ }^{8}$.

Por otra parte, en este medio se da una concentración de los usuarios más jóvenes y recalcitrantes del uso de Internet. Por lo que no podemos pensar que lo que veamos en este medio es representativo de lo que supone el colectivo de usuarios de Internet en extenso.

Al igual que en otros foros, existen multitud de redes de servidores para acceder a este medio de comunicación. En este caso adjunto un listado de canales abiertos un día cualquiera en la red hispana de servidores de IRC. A pesar de haber realizado una selección de los mismos, el listado es bastante largo, pero entiendo que su lectura es sumamente ilustrativa de lo que es este medio.

El nombre que aparece en primer lugar, situado junto al signo \#, es el nombre del canal, el número que aparece a continuación son las personas que en ese momento estaban conversando dentro del canal y la frase que hay en muchas ocasiones a continuación, es el topic o descripción ${ }^{9}$, comentarios, etc. colocados por los operadores del canal como elemento descriptivo o de invitación para sumarse al mismo.

GRUPOS DE IRC. En la red hispana.

pleyades.irc-hispano.org Fri Apr 24 12:52:41 1998

\#14-22gay 14 El CaNaL gAy Más jOvEn DeL iRc

\#accion-nacional 3 venid cabrones venid

\#adela 7 Quien vè el cielo en el agua, vè los peces en los árboles...!

\#ajedrez 7 ler torneo del Canal, inscríbete en: http://personal.redestb.es/poyete/

\#albacete 4 Se esta empezando a currar una web de canal para dar ideas escribir a dende_@geocities.com

8 Existe una abundante literatura sobre las guerras electrónicas en Internet, aunque los conflictos dentro de este contexto siempre se resuelven por la vía electrónica, en ocasiones puede tener importantes consecuencias para sus protagonistas, llegando a perder todo el contenido de los discos duros de su ordenador, etc.

${ }^{9}$ No he corregido errores ortográficos ni otro tipo de anomalías por motivos obvios. 
\#alcala_henares 9 _9,2\#H_Tha_os invita a su cumpleaños el finde del 25! Ella paga todo. (lalalaÆ) _

\#alcohólicos 3 øTienes problemas con el alcohol? øQuieres dejarlo y no lo consigues? Entra, quizás podamos ayudarte.

\#alicante 19 kedada el sabado a las 10 en el Mc o a la 1 en el Cure

\#almeria 4 4_ TOPIC has quit IRC__2 (Conecction reset by peer)

\#amigos_del_irc $3 \_2,7 \mathrm{Si}$ te va el buen rollo, te gusta hablar, entra te estamos esperando...

\#amistad_sincera $3<$ No dejes crecer la hierba en el camino de la amistad >

\#amor $94 \mathrm{La}$ injusticia de la vida es algo que cuestiono cada vez que pienso en la suerte que he tenido al conocerte \#madrid 52 \}|BYTEI\{ necesita urgentemente el PARTITION MAGIC 3

\#amor_sexo_lujuria 20 CANAL ORGASMIKO DEL IRC _LLEVAS LA MUDA LIMPIA?

\#amor_y_odio 4 buito esta destrozando a potter al guiñote y potter ya debe hasta los calzones

\#anarkia 5 Desde aki pido ayuda!!! a todos los ke apoyeis los movimientos revolucionaris de las guerrillas sudamericanas contactar con GuEvArA NUESTRO PUEBLO DEBE SER LIBR

\#argentina 5 Venf a tomar un mate cocido con galletas marineras...dale pas\$

\#artEs 3 _4,8CANAL ARTYS!!!!!!!!!!!!!!!!!!!!!!!!!!!!

\#athletic_club CANAL DEL ATHLETIC CLUB DE BILBAO 1898 - 1998

\#aula 8 PASA Y MIRA A ESPERTEYU SIN PANTALONES.

\#aviles4 Bienvenid@a Avilès

\#ayuda_irc 10 Entra y pregunta si tienes dudas de Irc o Mirc visita nuestra peich en http://www.arrakis.es/ -sucu

\#barÁa 4 _12Campions de lliga, i araFORZA JUVE

\#arcelona 23 <MIMAS> alucinamos con gente tan guay como vosotros!!!! <- Julio Posadas is my Idol

\#dsm 12 Canal de Sadomasoquismo en Castellano 18+

\#beatles 3 LOS BEATLES, yesterday, today and forever....here, there and everywhere....

\#beginner 38 http://nogue.home.ml.org Remodelada! Visitala! www.zoom.es/ beginner Pag. Del Canal

\#ierzo 3 Preparando ya la Kedada del 20 de Junio en Madrid.

\#bilbao 19 JAINKOA SE VA A ESKIAR

\#bisexuales 8 Para los que gustan de los dos sabores ;)))

\#olsa 27

\#bora-bora 5 Bienvenido a \#bora-bora

\#botica 4 FARMACIA DE GUARDIA _ $0,0 \_0,3$ CONSULTE CON SU FARMĀCEUTICO/A

\#raguitas 4

\#bros 3 _8,2EEEEECanal del Buen Rollo y Mejor Gente!!!!!

\#burgoscity 3 _4,1\#_B_u_RG_o_SC_i_T_y e_S K_a_ST_i_LL_a _Y L_o D_e_M_a_S_e_S T_ie_RR_a

\#Cádiz 6 "VEN A CADIZ, PRONTO LLEGARA EL VERANO!!!"

\#camelot 15 La Reina nos honra con su presencia hoy

\#canal_cristiano 4 "Jesucristo es el mismo ayer, hoy y siempre. Heb 13,8"

\#canal_satanico 12 _12_KRILIN ES MAS BUJA KE NINGUNO0000000, JURJURJUR

\#canarias 23 Cuidado estamos ya en PRIMAvera, empiezan las cosquillas\# 
\#cannabis 11 amonal la PuNkArrA eStA En eL KaNaL!!!!!!!

\#cantabria_gold 3 Bienvenido a \#cantabria_gold, øque mas quieres?

\#carballinho 8

\#carmageddon 4 BIENVENIDO AL CANAL MAS SANGRIENTO DE LA HISTORIA... EL CANAL DEL CARMAGEDDON!!!!!!!!

\#Catalunya 59 Miau miau 19 petons amb llengua(la meva edat) jo tmb en vull! \#cinefilos 17 New le dijo ayer antipatica a dua, ya macueldo :)

\#ciudad_real 5 el dia 30 gran makrokedad del kanal, no faltes, si piensas venir escribe a (lo ke@sea)

\#contactos_sexuales 16 EN EL PAIS DE LAS MARAVILLASEEE

\#CoruÑa 10 SE VENDE STAR CRAFT VERSION FULL CD POR 1.400

\#cyber_punk 5_11,1[[[_7,1—_[-_11,1[www.ibizasun.com/cyberpunk]

\#cybermus 3 Entra y disfruta de este maravilloso juego con nosotros

\#darknight $8^{\wedge \wedge} \wedge \sim^{*}+., B^{\wedge} \wedge \wedge \sim^{*}+. .$, DaRkNiGhT FoReVeR $\wedge^{\wedge} \wedge \sim^{*}+. ., B^{\wedge} \wedge \sim^{*}+$.

\#De25a35 18 Geisha's Personal Web Page @_http://www.izar.net/ geisha/_

\#deprimidos 10 Tenemos emision de radio:Domingos 14 a $16 \mathrm{~h}$ en 96.3 Barcelona.

\#dj's 9 http://www2.infotelecom.es/ unlock/

\#donosti 6 http://come.to/donosti - P\$gina de USUARI@S actualizada

\#dracula 6 Es eso todo lo que sabes hacer por mi ? .... cuanto esperas y ahora que no llamas ...

\#el_refugio 7 \#El_refugio te ofrece su cobijo http://www.arrakis.es/ ajimeno/el_refugio.htm

\#el_rincon 7 Visita la pagina del Rincon : members.xoom.com/el_rincon

\#ElCastillo 25 tonny es una persona de honor intachable, el no ha nukeado a nadie

\#españa 10 ENTRA Y HABLA DE LO QUE QUIERAS.... MENOS DE POLITICA

\#euskadi 14

\#Extremadura El canal amigo de toda_4,8 ESPAÑA _

\#fotos_xxx 23 http://www.netspain.com/ fireice

\#gai 4 Aiers busca a bcn-chip desesperadamente

\#galicia 37

\#gay 16 Animaros a entrar... la fiesta esta aqui dentro...

\#gay_andalucia
www.geocities.com/WestHollywood/Village/8505

CON PAGINA:

\#gaybcn 12 Aiers busca a bcn-chip desesperadamente

\#gaycanarias $6 \mathrm{El}$ de siempre...

\#gayfotos 4

\#gaymadrid 11 Entra en el canal más GuAY de Madrid...

\#gaypajas 13 Bienvenido al canal de los gays con más morbo de todo internet. Si

quieres nivel un mail a devil@ufoplanet.com o query a Iron

\#gaysm 5 De Amos, esclavos y similares.

\#gayvalencia 6 Bienvenido a \#gayvalencia

\#granada 42 VIOLATOR BOLLITO DE CREMA DE POCHOLATE

\#greenpeace $4 \quad 3$ ENTRAR EN \#irc_musica SE ESTA REGISTRANDOOOOOOO

\#grunge 6 NO KEREMOS A SCYTALE !!!!! manda un maila delfin@ delqui.us.es para desregistrar

\#guitarra 3 porfavoir enviad fotos pa la g_eb!!!!!!!!!!!!! 
\#hacker 5 //HacK To LiVe \& LiVe To HacK \#ayuda_para_novatos \#HaRDCoRe!!! 4

\#hip-hop 3 comunicologia en gira dia 24 en madrid sala macumba,

\#insultos 12 [11:51] <phenix> no te amenazo ya eres historia

\#irc_musica 7 PASAR Y CAMBIAR MIDIS Y WAVs_\#irlanda 4 IRLANDA:la bella dama verde

\#a_kloaka 4 _9,1VIVE Y DEJA FUMAR

\#la_plaza 9 Beli, Pima y Arse, se van de juerga esta Noche, Quien diablos se APUNTA??

\#leon4-=_@ \#leon@-

\#lesb_ops 4 Canal de ayuda a \#lesbianas. Para consejos sentimentales acudir a Elena Francissss ....

\#lesbianas 20 Canal de Mujeres que Aman a Mujeresłmp3 20 Bienvenidos al canal

\#mp3 - El Templo de la Musica

\#lesbianitas 4 canal de mujercitas

\#linux 21 http://linux.compusis.com $\rightarrow$ esta abierto el plazo de peticion de registros.

\#lleida 3

\#logroÑo 9 8=====0- - . . - SIIIIIIIIIII NECROZOOFILIAAAAAA -

\#lorca 3 Ciudad del Sol

\#macintosh 4 KEDADA EN MADRID 2 DE MAYO. +

\#magisterio 3

\#malaga 19 Esta noche, como siempre, quedada en el Bauhaus a partir de las 22.30

\#malal 5 LA M.A.L.A.L.T.I.A TY UN NOM: MUSIQUE

\#mallorca 3 \#Topic No Set\#Topic Nule\#

\#malos 6 Muchas gracias a la gente de este canal que me ha ayudado mazo... :***** asias

\#más_de_30 35 Altair, si llegas, espera a Magnolia!!!!!!!!!!!!!!!

\#Más_de_40 14 Bienvenido a \#Más_de_40

\#mas_sevilla $10 \mathrm{KFE}$ : Tranki Ryoguin, to es koÑa, es k no me konoces??

\#medicina 6 EEECanal de MĖdicos, Cirujanos y algùn que otro PACIENTE!!!

\#midi-chat 12 8MiDiS_15,1 http://www.internetw.net/0,1y_9,1http://www. musica.org/midilandia

\#moteros 4 bienvenido a \#moteros ..la gueb es www.geocities.com/motorcity/ 56741

\#murcia 74 La vida es una mierda, y si no cuidamos los pequeños detalles, pues nos pueden ir dando

\#haturismo 6 Desnudos lo más Natural

\#necropage $9 \mathrm{http}: / /$ members.xoom.com/necropage ONE WORLD UNDER SATAN!

\#opers_help 10 _Ayuda de IRC Hispano_. Pregunta por query a un operador. \#orlando 4 ESPÁSIO DE MUJERES PATROSINADO POR BULL \#ourense 17 BIENVENIDOS AL CANAL DE LA FIESTA JOLGORRIO!!!!! \#poesia QUEDADA DEL CANAL DE POESIA EN MADRID, El viernes 24 de Julio EEE NO FALTES A LA CITA CON LOS POETAS !!!

\#pontevedra 7 http://www.geocities.com/SouthBeach/Breakers/2344

\#programacion 5

\#psiquiatrico 8 Bienvenido a \#psiquiatrico 
\#Punk 4 Un PaTrIoTa.... uN iDiOtA!!!!

\#puta_espaÑa 5 MUERTE A LA PUTA ESPACAEEEEEEEEEE

\#putos_fatxas 7 muerte a los fascistas espaÑolesEE

\#rol 9 Club de rol. pregunta por aqui, y te informan guay la gente esta de paz

\#romanticos Un comienzo no desaparece nunca, ni siquiera con un final ...

\#salamanca 8 Para cuando la _4,8MACROKEDADA???????

\#Santiago 6 Clampostela, Clantera .. se buscan fundas para balas !!

\#Santiago_Compostela 16 Bienvenido a \#santiago_compostela

\#sevilla 18 _ 4KEDADA GENERAL DE IRC DIA 24 en SEVILLA...

\#sexo 72

\#sexo_casadas 9

\#snowboard 4 _9,6Snowboard Is Life!!!!. _ 9,2Visitanos en: _15http://surf.to/ snowboard

\#soziedad_alkoholika 3 _ 0,10 (S.A.)_9,10 La Kosa No Esta Komo p-a Tirar Kohetes

\#sumisas 16

\#tierra_media 3 Quien tenga un Silmaril que lo devuelva...(Los hijos de Feanor)

\#torremolinos 3 Bienvenidos a Torremolinos, Paraiso Terrenal, Visita Nuestra web :www.vegasicilia.com

\#trivial 30 FELICIDADES RULO POR TU EMBARAZO

\#universidad 14 _8,6 Entra y pasalo bien en el canal joven mas entretenido de IRC !! ;P

\#ups 3 Universidad Pontificia de Salamanca en Madrid

\#valencia 22 FIESTA! ALEGRIA! CON LA PELBIS ARRIBA Y SONRIENDO!!

\#vampiro 16 QUEDADA EN JULIO EN SANTANDER LOS DIAS 8-15 DE JULIO

\#vitoria 13 !Mecag_endios! !Que cuadrilla!

\#webcafe-granada 8 El Unico Sitio Del Mundo Donde El Cafe Es Algo Mas Que Una Tertulia

\#zanatorio 3 MaTa A un0s PokOz y SerAS un AZeSino Mata a uno MiLLOnS y SerAZ un KonKizTAdoR

\#zaragoza 25 COMIDA CAMPESTRE EL DOMINGO A LA 1:15 EN EL PARQUE GRANDE

\section{Lista de correo}

Las listas de correo, como ya comenté anteriormente, son redistribuidores de correo electrónico sobre un tema concreto. Son la herramienta más práctica de Internet para mantenerse informado sobre un tema concreto. A veces, suponen un "peligro» por su eficacia, ya que existen listas tan activas y con tantos integrantes que producen una cantidad de información diaria respecto al tema tratado muy difícil de asimilar. A pesar de ésto siempre es recomendable estar suscrito a las listas que nos interesen, sobre todo profesionalmente, ya que además de la información pueden proporcionar contactos personales y colaboradores sobre materias comunes. 
La mecánica de funcionamiento de las listas de correo es sumamente sencilla al estar basado en el correo electrónico. En este sentido, lo único que es necesario tener claro es la forma de suscripción (y de darse de baja) a la misma y las reglas internas de dicha lista, que se comunicarán en el momento de inscribirse.

Como comenté anteriormente, las listas de correo son los foros más serios y es recomendable antes de participar en los mismos, leer las normas de uso y asistir al cruce de unas cuantas decenas de mensajes como mínimo.

A continuación se expone un listado de éstos foros, de interés para la investigación en ciencias sociales y complementarias a ésta ${ }^{10}$.

\section{Listas de correo en castellano}

ambiental@listserv.rediris.es

políticas de protección y desarrollo sostenible

cde-es@listserv.rediris.es

Foro sobre la Unión Europea y España

cederul@listserv.rediris.es

Foro sobre desarrollo rural

colon@listserv.rediris.es

Historia y tecnologías de la información

diseven@listserv.rediris.es

Informacion de Congresos,Jornadas,Seminarios etc

demogr-h@listserv.rediris.es

Poblacion e Historia Economica

docbd@listserv.rediris.es

Documentación en la Universidad española

edudist@listserv.rediris.es

educación a distancia

etnoedu@listserv.rediris.es

investigación Etnográfica en educación

grumetes@listserv.rediris.es

Foro para principiantes en Internet

herm@listserv.rediris.es

Banco de Datos y Ciencias Humanas

${ }^{10}$ Podemos encontrar esta lista ampliada y actualizada en: http://www.rediris.es/list/ 
info-europa@listserv.rediris.es

Boletin electronico sobre la Union Europea

lapepa@listserv.rediris.es

Historia contemporanea de España

ofistat@listserv.rediris.es

Foro para productores y usuarios de estadística. Oficial en

España

periodismo@listserv.rediris.es

Periodismo y medios de comunicacion en castellano

servinfo@listserv.rediris.es

Servicios de información universitarios

ant-arq@ccc.uba.ar

Lista de intercambio de Antropologia y Arqueologia

antropol-list@etsiig.uniovi.es

Foro general sobre antropologia

araca@ccc.uba.ar

Estudios de género y estudios de la mujer

cts-list@etsiig.uniovi.es

Filosofía sobre Ciencia, tecnología y Sociedad

fcr-h@recerca.fcr.es

información de Becas y Ayudas de Ciencias Sociales y Ciencias Humanas

fcr-int@recerca.cfr.es

información de becas internacionales de fuentes de financiación para la investigación

grround@tinet.fut.es

CiberTertulia Grround

infoworkers@ung.edu.ar

Trabajadores de la información en el nuevo milenio

1-ecolog@cicei.ulpgc.es

Foro sobre ecologismo y desarrollo sostenible

li@foros.bcsnetwork.es

Libertad en Internet

ml-uned@kaos.es

Lista de los estudiantes de la UNED

openmkt@ictnet.es

Marketing, publicidad y comunicación on-line 
tciencia-list@etsiig.uniovi.es

Teoria y filosofía de las Ciencias

utopia@dis.ulpgc.es

Discusiones sobre ideologías progresistsa y modelos sociales en general.

\section{BIBLIOGRAFÍA DE APROXIMACIÓN A LA INTERNET COMO OBJETO SOCIAL}

AUTORES VARIOS (1997): «Informática, información y comunicación». Documentación social. N. ${ }^{\circ} 108$. Jul B Sept.

BERICAT, E. (1996): «La sociedad de la información. Tecnología, cultura, sociedad». REIS. $n .^{\circ} 76$, pp. 99-121.

CASTELLS, M. (1997): La era de la información. Economía, sociedad y cultura. Vol 1. La sociedad red, Madrid: Alianza Editorial.

Coupland, D. (1996): Microsiervos, Barcelona: Ediciones B.

Dyson, E. (1997): Release 2.0. Barcelona: Ediciones B.

ECHEVERRIA, J. (1994): Telépolis, Madrid: Ensayos/Destino.

- (1995): Cosmopolitas domésticos, Barcelona: Anagrama.

NeGroponte, N. (1995): El mundo digital, Barcelona: Ediciones B.

Piscrtell, A. (1995): Ciberculturas en la era de las máquinas inteligentes, Barcelona: Paidos,

Quéau, Ph. (1995): Lo virtual. Virtudes y vértigos, Barcelona: Paidós Hipermedia.

RHEINGold, H. (1996): A slice of my life in my virtual community. en Godwin. Mike. High Noon on the Electronic Frontier. Londres: MIT.

TREJO DeLARBRE, R. (1996): Nueva alfombra mágica: usos y mitos de Internet, la red de redes. Madrid: Fundesco.

WoOlley, B. (1994): El universo virtual, Madrid: Ed. Acento. 